\title{
Jeffrey Fluid Flow through Porous Medium in the Presence of Magnetic Field in Narrow Tubes
}

\author{
Santhosh Nallapu and G. Radhakrishnamacharya \\ Department of Mathematics, National Institute of Technology, Warangal 50604, India \\ Correspondence should be addressed to Santhosh Nallapu; princenallapu@gmail.com
}

Received 13 February 2014; Accepted 4 June 2014; Published 25 June 2014

Academic Editor: George S. Dulikravich

Copyright ( $) 2014$ S. Nallapu and G. Radhakrishnamacharya. This is an open access article distributed under the Creative Commons Attribution License, which permits unrestricted use, distribution, and reproduction in any medium, provided the original work is properly cited.

\begin{abstract}
Jeffrey fluid flow in the presence of magnetic field through porous medium in tubes of small diameters is studied. It is assumed that the core region consists of a Jeffrey fluid and the peripheral region of a Newtonian fluid. Making the assumptions as in the work of Chaturani and Upadhya, the linearised equations of motion have been solved and analytical solution has been obtained. The influence of various pertinent parameters on the flow characteristics such as effective viscosity, core hematocrit, and mean hematocrit has been studied and discussed through graphs. It is found that the effective viscosity and mean hematocrit decrease with Jeffrey parameter and Darcy number but increase with tube hematocrit and tube radius. Also, the core hematocrit decreases with Jeffrey parameter, Darcy number, tube hematocrit, and tube radius. Further, it is noticed that the flow exhibits the anomalous Fahraeus-Lindquist effect.
\end{abstract}

\section{Introduction}

Microcirculation is a part of human circulatory system, which consists of a complex network of blood vessels, whose diameter ranges from approximately $20 \mu \mathrm{m}$ (microns) to $500 \mu \mathrm{m}$. There are several types of vessels in microcirculation such as arterioles, capillaries, and venules. Its main functions are to supply oxygen and nutrients to every part of the human body. Several anomalous effects have been observed in microcirculation. In particular, the apparent viscosity of the blood increases with tube diameter and this is referred to as the Fahraeus-Lindquist effect. This effect has been confirmed by several investigators.

To explain the observed Fahraeus-Lindquist effect, Haynes [1] considered a two-fluid model with both fluids as Newtonian fluids with different viscosities. Bugliarello and Sevilla [2] have considered a two-fluid model where in the core region as well as peripheral region fluids are both Newtonian with different viscosities or both fluids are Casson's fluid with different yield coefficients and viscosities. Sharan and Popel [3] and Srivastava [4] have reported that, for blood flowing through narrow tubes, there is a peripheral layer of plasma and a core region of suspension of all erythrocytes. Following the theoretical study of Haynes [1] and experimentally tested model of Bugliarello and Sevilla [2], two-fluid modeling of blood flow has been discussed and used by a good number of researchers. Several nonNewtonian fluid models have been considered for blood flow in small diameter tubes. Chaturani and Upadhya [5, 6] analyzed two-fluid models assuming Newtonian fluid in peripheral region and micropolar and couple stress fluids in the core region. Bali and Awasthi [7] presented a mathematical model for blood flow in a small blood vessel in the presence of a magnetic field. Kumar et al. [8] investigated a computational technique for flow in blood vessels with porous effects. Gupta [9] investigated computational study of blood flow through stenosed artery with magnetic effects.

A porous medium is a material volume consisting of solid matrix with an interconnected void. Flows through a porous medium have several practical applications present in nature: flow in sand beds, in petroleum reservoir rocks, slurries, sedimentation, and so forth. Examples of natural porous 
media are beach sand, sandstone, limestone, the human lung, bile duct, and gall bladder with stones in small blood vessels. Flow through porous medium has been studied by a number of workers employing Darcy's law. Furthermore, the study of magnetohydrodynamics (MHD) flow problems has gained considerable interest because of its extensive engineering and medical applications. The MHD deals with the dynamics of electrically conducting fluids. Some investigators have considered the MHD studies of Newtonian and non-Newtonian fluids in different flow geometries. Misra et al. [10] have investigated a mathematical modeling of blood flow in porous vessel having double stenosis in the presence of an external magnetic field. Eldabe et al. [11] studied the peristaltic motion of non-Newtonian fluid with heat and mass transfer through a porous medium in the channel under the effect of magnetic field.

A non-Newtonian fluid model that has attracted many researchers is the Jeffrey fluid as this is found to be a better model for physiological fluids [12]. Jeffrey fluid model is a significant generalization of Newtonian fluid model as the latter one can be deduced as a special case of the former. Several researchers have studied Jeffrey fluid flows under different conditions. Ebaid et al. [13] have studied the peristaltic transport in an asymmetric channel through a porous medium. Vajravelu et al. [14] investigated the influence of heat transfer on peristaltic transport of a Jeffrey fluid. Jyothi et al. [15] have considered the pulsatile flow of a Jeffrey fluid in a circular tube lined internally with porous material. Ebaid [16] has analyzed a mathematical model to study the peristaltic transport of an incompressible viscous fluid in an asymmetric channel under the effect of transverse magnetic field with slip boundary conditions. Akbar et al. [17] have studied the Jeffrey fluid model for the peristaltic flow of chyme in the small intestine with magnetic field. Abd-Alla et al. [18] have investigated the peristaltic flow of a Jeffrey fluid in an asymmetric channel. Recently, Nallapu and Radhakrishnamacharya [19] studied a two-fluid model for the flow of Jeffrey fluid in tubes of small diameters.

In the present paper, a two-fluid model for the flow of Jeffrey fluid through a porous medium in tubes of small diameters with magnetic effect has been investigated. It is assumed that the core region consists of Jeffrey fluid and the peripheral region consists of Newtonian fluid. Making the assumptions as in the work of Chaturani et al. [6], the linearised equations of motion have been solved and analytical solution has been obtained. The influence of various pertinent parameters on the flow characteristics such as effective viscosity, core hematocrit, and mean hematocrit has been studied.

\section{Formulation of the Problem}

Consider an axisymmetric, laminar, steady flow of an electrically conducting Jeffrey fluid through a circular tube of radius " $a$ " filled with porous medium. It is assumed that a uniform magnetic field $B_{0}$ is applied transversely to the flow. It is assumed that the system consists of two immiscible layers of fluids; the inside layer is non-Newtonian fluid obeying

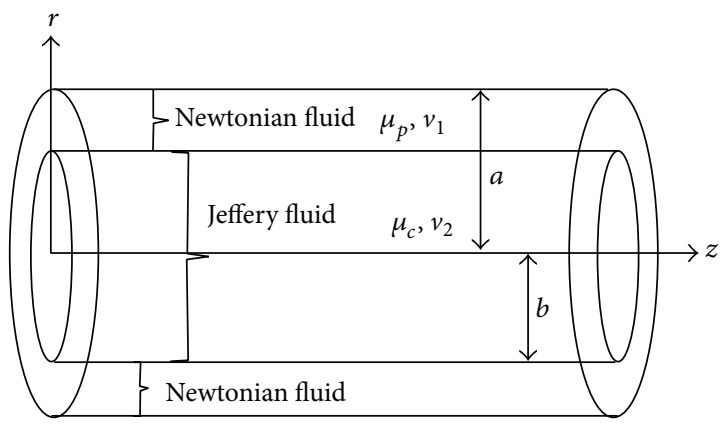

FIgURE 1: Flow geometry of blood in small vessels.

Jeffery model and takes cylindrical shape of radius $b$, while the outside layer is Newtonian fluid and takes cylindrical shape of radius $a$. Further, the cylinders have a common axis. It is clear that the surface of discontinuity between them is imaginary due to the two fluids being immiscible, as shown in Figure 1. Let $\mu_{p}$ and $\mu_{c}$ be the viscosities of Newtonian fluid in the peripheral region and Jeffrey fluid in the core region, respectively.

Here, $\bar{q}$ is the velocity of the fluid, $\bar{j}$ is the current density, $\bar{B}=\left(\bar{B}_{0}+\bar{B}_{1}\right)$ is the total magnetic field, $\bar{B}_{1}$ is the induced magnetic field $\left(\bar{B}_{1} \ll \bar{B}_{0}\right)$, and $\bar{j} \times \bar{B}$ is Lorentz's force which is the body force acting on the fluid. The Maxwell equations and Ohm's law (on neglecting the displacement currents) are

$$
\begin{aligned}
& \nabla \cdot \bar{B}=0, \quad \nabla \times \bar{B}=\mu_{m} \bar{j}, \quad \nabla \times \bar{E}=-\frac{\partial \bar{B}}{\partial t}, \\
& \bar{j}=\sigma(\bar{E}+\bar{q} \times \bar{E}),
\end{aligned}
$$

where $\sigma$ is the electrical conductivity, $\mu_{m}$ is the magnetic permeability, and $\bar{E}$ is the electric field. The imposed and induced electric fields are assumed to be negligible. The magnetic Reynolds number is assumed to be small so that the induced magnetic field is neglected.

Hence, the force $\bar{j} \times \bar{B}$ simplifies to

$$
\bar{j} \times \bar{B}=-\sigma B_{0}^{2} w .
$$

Cylindrical polar coordinate system $(r, \theta, z)$ is chosen, where the $z$-axis is taken along the axis of the tube. The equations governing the steady two-dimensional flow of an incompressible Jeffrey fluid for the present problem are as follows.

Equation of Continuity. One has

$$
\frac{\partial v_{r}}{\partial r}+\frac{v_{r}}{r}+\frac{\partial v_{z}}{\partial z}=0 .
$$

Equations of Motion. Consider

$$
\begin{aligned}
& \rho\left[v_{r} \frac{\partial}{\partial r}+v_{z} \frac{\partial}{\partial z}\right] v_{r} \\
& =-\frac{\partial p}{\partial r}+\frac{1}{r} \frac{\partial}{\partial r}\left(r \bar{S}_{r r}\right)+\frac{\partial}{\partial z}\left(\bar{S}_{r z}\right)-\frac{\mu_{c}}{k_{0}} v_{r}
\end{aligned}
$$




$$
\begin{aligned}
& \rho\left[v_{r} \frac{\partial}{\partial r}+v_{z} \frac{\partial}{\partial z}\right] v_{z} \\
& \quad=-\frac{\partial p}{\partial z}+\frac{1}{r} \frac{\partial}{\partial r}\left(r \bar{S}_{z r}\right)+\frac{\partial}{\partial z}\left(\bar{S}_{z z}\right)-\sigma B_{0}^{2} v_{z}-\frac{\mu_{c}}{k_{0}} v_{z}
\end{aligned}
$$

in which

$$
\begin{aligned}
& \bar{S}_{r r}=\frac{2 \mu_{c}}{1+\lambda_{1}}\left[1+\lambda_{2}\left(v_{r} \frac{\partial}{\partial r}+v_{z} \frac{\partial}{\partial z}\right)\right]\left(\frac{\partial v_{r}}{\partial r}\right), \\
& \bar{S}_{r z}=\bar{S}_{z r}=\frac{\mu_{c}}{1+\lambda_{1}}\left[1+\lambda_{2}\left(v_{r} \frac{\partial}{\partial r}+v_{z} \frac{\partial}{\partial z}\right)\right]\left(\frac{\partial v_{z}}{\partial r}+\frac{\partial v_{r}}{\partial z}\right), \\
& \bar{S}_{z z}=\frac{2 \mu_{c}}{1+\lambda_{1}}\left[1+\lambda_{2}\left(v_{r} \frac{\partial}{\partial r}+v_{z} \frac{\partial}{\partial z}\right)\right] \frac{\partial v_{z}}{\partial z}
\end{aligned}
$$

are the extra stress components.

Further, $v_{r}$ and $v_{z}$ are the velocity components in the $r$ and $z$-directions, respectively, $\lambda_{1}$ is the ratio of relaxation to retardation times, $\lambda_{2}$ is the retardation time, $p$ is the pressure, $\rho$ is the density, $k_{0}$ is the permeability of the porous medium, $\sigma$ is the electrical conductivity of the fluid, $\mathrm{Da}\left(=k_{0} / a^{2}\right)$ is the Darcy number, and $M\left(=\sqrt{\sigma / \mu} B_{0} a\right)$ is the magnetic parameter.

It is assumed that the flow is in the $z$-direction only and hence the velocity component $v_{r}=0$. Consequently, the equations governing the flow of fluid (Jeffrey fluid) in the core region $(0 \leq r \leq b)$ reduce to

$$
\begin{gathered}
\frac{\partial p}{\partial r}=0 \\
\frac{\mu_{c}}{1+\lambda_{1}} \frac{1}{r} \frac{\partial}{\partial r}\left(r \frac{\partial v_{z}}{\partial r}\right)-\sigma B_{0}^{2} v_{z}-\frac{\mu_{c}}{k_{0}} v_{z}-\frac{\partial p}{\partial z}=0 .
\end{gathered}
$$

Let $v_{z}(r)=v_{1}(r)$ be the velocity in the peripheral region and $v_{2}(r)$ in the core region. The equations governing the flow of fluid are as follows.

\section{Peripheral Region (Newtonian Fluid). Consider}

$$
\mu_{p} \frac{1}{r} \frac{\partial}{\partial r}\left(r \frac{\partial v_{1}}{\partial r}\right)-\sigma B_{0}^{2} v_{1}-\frac{\mu_{p}}{k_{0}} v_{1}-\frac{\partial p}{\partial z}=0
$$

$$
\text { for } b \leq r \leq a .
$$

Core Region (Jeffrey Fluid). One has

$$
\begin{aligned}
& \frac{\mu_{c}}{1+\lambda_{1}} \frac{1}{r} \frac{\partial}{\partial r}\left(r \frac{\partial v_{2}}{\partial r}\right)-\sigma B_{0}^{2} v_{2}-\frac{\mu_{c}}{k_{0}} v_{2}-\frac{\partial p}{\partial z}=0 \\
& \text { for } 0 \leq r \leq b \text {, }
\end{aligned}
$$

where $\partial p / \partial z$ is the constant pressure gradient.

The boundary conditions for the problem are given by

$$
\begin{gathered}
v_{1}=0, \quad \text { at } r=a, \\
v_{1}=v_{2}, \quad \tau_{1}=\tau_{2}, \quad \text { at } r=b, \\
v_{2} \text { is finite at } r=0 .
\end{gathered}
$$

Condition (9a) is the classical no-slip boundary condition for the velocity; (9b) denotes the continuity of velocities and stresses at the interface and (9c) is the regularity condition.

Solving (7) and (8) under conditions (9a), (9b), and (9c), we get

$$
\begin{array}{r}
v_{1}(\xi)=\frac{a^{2} P}{\mu_{p} \alpha}\left(1-\frac{I_{0}(\sqrt{\alpha} \cdot \xi)}{I_{0}(\sqrt{\alpha})}\right) \quad \text { for } d \leq \xi \leq 1, \\
v_{2}(\xi)=\frac{a^{2} P}{\mu_{p} \alpha}\left(1-\frac{I_{0}(\sqrt{\alpha} \cdot d)}{I_{0}(\sqrt{\alpha})}+\mu^{\prime} \beta\left[1-\frac{I_{0}(\omega \cdot \xi)}{I_{0}(\omega d)}\right]\right) \\
\text { for } 0 \leq \xi \leq d,
\end{array}
$$

where

$$
\begin{aligned}
\xi & =\frac{r}{a}, \quad d=\frac{b}{a}, \quad P=-\frac{\partial p}{\partial z}, \\
\mu^{\prime} & =\frac{\mu_{p}}{\mu_{c}}, \quad \alpha=M_{p}^{2}+\frac{1}{\mathrm{Da}}, \\
\beta & =\frac{\alpha}{M_{c}^{2}+(1 / \mathrm{Da})}, \\
\omega & =\sqrt{\left(1+\lambda_{1}\right)\left(M_{c}^{2}+\frac{1}{\mathrm{Da}}\right)} .
\end{aligned}
$$

The flow flux in the peripheral region, denoted by $Q_{p}$, is defined by

$$
Q_{p}=2 \pi a^{2} \int_{d}^{1} v_{1}(\xi) \xi d \xi
$$

Substituting for $v_{1}(\xi)$ from (10) in (13), we get

$$
Q_{p}=\frac{a^{4} P \pi}{\mu_{p} \alpha}\left(1-d^{2}+2 \frac{d I_{1}(\sqrt{\alpha} \cdot d)-I_{1}(\sqrt{\alpha})}{\sqrt{\alpha} I_{0}(\sqrt{\alpha})}\right) .
$$

Similarly, the flow flux in the core region is given by

$$
\begin{aligned}
Q_{c} & =2 \pi a^{2} \int_{0}^{d} v_{2}(\xi) \xi d \xi \\
& =\frac{a^{4} P \pi}{\mu_{p} \alpha}\left(d^{2}-d^{2} \frac{I_{0}(\sqrt{\alpha} \cdot d)}{I_{0}(\sqrt{\alpha})}+\mu^{\prime} \beta\left[d^{2}-2 d \frac{I_{1}(\omega d)}{\omega I_{0}(\omega d)}\right]\right) .
\end{aligned}
$$

Thus, the flow flux through the tube is given by

$$
Q=Q_{p}+Q_{c} .
$$

Using (14) and (15) in (16), we get

$$
\begin{gathered}
Q=\frac{a^{4} P \pi}{\mu_{p} \alpha}\left(1+2 \frac{d I_{1}(\sqrt{\alpha} \cdot d)-I_{1}(\sqrt{\alpha})}{\sqrt{\alpha} I_{0}(\sqrt{\alpha})}-d^{2} \frac{I_{0}(\sqrt{\alpha} \cdot d)}{I_{0}(\sqrt{\alpha})}\right. \\
\left.+\mu^{\prime} \beta\left[d^{2}-2 d \frac{I_{1}(\omega d)}{\omega I_{0}(\omega d)}\right]\right) .
\end{gathered}
$$


Comparing (17) with flow flux for Poiseuille flow, we get the effective viscosity as follows:

$$
\begin{aligned}
& \mu_{\mathrm{eff}} \\
& =\mu_{p} \alpha \times\left(8 \mathrm { Da } \left(1+2 \frac{d I_{1}(\sqrt{\alpha} \cdot d)-I_{1}(\sqrt{\alpha})}{\sqrt{\alpha} I_{0}(\sqrt{\alpha})}\right.\right. \\
& -d^{2} \frac{I_{0}(\sqrt{\alpha} \cdot d)}{I_{0}(\sqrt{\alpha})} \\
& \left.\left.+\mu^{\prime} \beta\left[d^{2}-2 d \frac{I_{1}(\omega d)}{\omega I_{0}(\omega d)}\right]\right)\right)^{-1} .
\end{aligned}
$$

2.1. Mean Hematocrit for Cell-Free Wall Layer. The percentage volume of red blood cells is called the hematocrit and is approximately $40-45 \%$ for human adults.

The core hematocrit $H_{c}$ is related to the hematocrit $H_{0}$ of blood leaving or entering the tube by

$$
H_{0} Q=H_{c} Q_{c}
$$

Substituting for $Q_{c}$ and $Q$ from (15) and (17) in (19), we get (after simplification)

$$
\begin{aligned}
& \bar{H}_{c} \\
& =\frac{H_{c}}{H_{0}}=1 \\
& +\left(1-d^{2}+2 \frac{d I_{1}(\sqrt{\alpha} \cdot d)-I_{1}(\sqrt{\alpha})}{\sqrt{\alpha} I_{0}(\sqrt{\alpha})}\right. \\
& \left.\quad \times\left(d^{2}-d^{2} \frac{I_{0}(\sqrt{\alpha} \cdot d)}{I_{0}(\sqrt{\alpha})}+\mu^{\prime} \beta\left[d^{2}-2 d \frac{I_{1}(\omega d)}{\omega I_{0}(\omega d)}\right]\right)^{-1}\right),
\end{aligned}
$$

where $\bar{H}_{c}$ is the normalized core hematocrit.

The mean hematocrit within the tube $H_{m}$ is related to the core hematocrit $H_{c}$ by

$$
H_{m} \pi a^{2}=H_{c} \pi b^{2}
$$

On simplification, we get

$$
\bar{H}_{m}=\frac{H_{m}}{H_{0}}=\frac{H_{c}}{H_{0}} d^{2}=\bar{H}_{c} d^{2},
$$

where $\bar{H}_{m}$ is the normalized mean hematocrit.

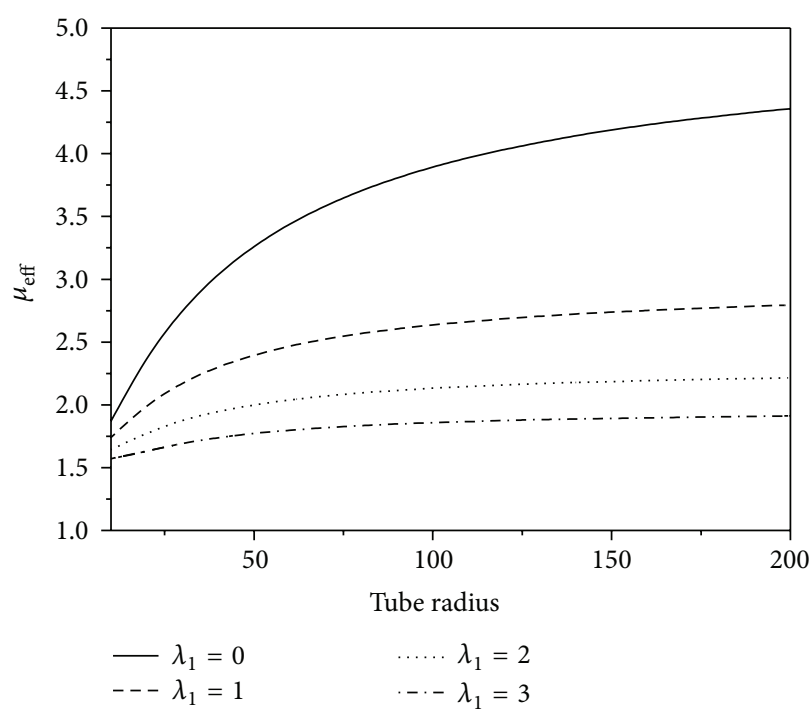

FIGURE 2: Effect of $\lambda_{1}$ on $\mu_{\text {eff }}\left(H_{0}=40 \%, M_{p}=1, M_{c}=0.5\right.$, and Da $=0.5$ ).

Substituting for $\bar{H}_{c}$ from (20) in (22), we get

$$
\begin{aligned}
& \bar{H}_{m}= \frac{H_{m}}{H_{0}}=\frac{H_{c}}{H_{0}} d^{2} \\
&=\left(1+\left(1-d^{2}+2 \frac{d I_{1}(\sqrt{\alpha} \cdot d)-I_{1}(\sqrt{\alpha})}{\sqrt{\alpha} I_{0}(\sqrt{\alpha})}\right.\right. \\
& \quad \times\left(d^{2}-d^{2} \frac{I_{0}(\sqrt{\alpha} \cdot d)}{I_{0}(\sqrt{\alpha})}\right. \\
&\left.\left.\left.\quad+\mu^{\prime} \beta\left[d^{2}-2 d \frac{I_{1}(\omega d)}{\omega I_{0}(\omega d)}\right]\right)^{-1}\right)\right) d^{2} .
\end{aligned}
$$

\section{Results and Discussion}

In order to discuss the effects of Jeffrey parameter $\left(\lambda_{1}\right)$, core magnetic parameter $\left(M_{c}\right)$, Darcy number $(\mathrm{Da})$, tube hematocrit $\left(H_{0}\right)$, and tube radius $(a)$ on effective viscosity $\mu_{\text {eff }}$, core hematocrit $\bar{H}_{c}$, and mean hematocrit $\bar{H}_{m}$, they have been numerically computed and graphically presented in Figures 2-13. In the present analysis, the values of $\mu_{p}$ are taken as $1.2 \mu \mathrm{m}, \mu_{c}=4.0 \mu \mathrm{m}$ and the value of $d$ is calculated from the relation $d=1-(\epsilon / a)$ in which $\epsilon=3.12 \mu \mathrm{m}$ for $40 \%$ hematocrit, $3.60 \mu \mathrm{m}$ for $30 \%$, and $4.67 \mu \mathrm{m}$ for $20 \%$ [5].

Figures $2-5$ show the variation of effective viscosity $\left(\mu_{\text {eff }}\right)$ for different values of Jeffrey parameter $\lambda_{1}$, core magnetic parameter $\left(M_{c}\right)$, Darcy number $(\mathrm{Da})$, tube hematocrit $\left(H_{0}\right)$, and tube radius $(a)$. It can be seen that the effective viscosity decreases with Jeffrey parameter $\left(\lambda_{1}\right)$ (Figure 2) and Darcy number (Da) (Figure 3) but increases with core magnetic parameter $\left(M_{c}\right)$ (Figure 4 ) and tube hematocrit $H_{0}$ (Figure 5). These results are in agreement with the results of Bugliarello and Sevilla [2], Srivastava [4], and Chaturani and Upadhya 


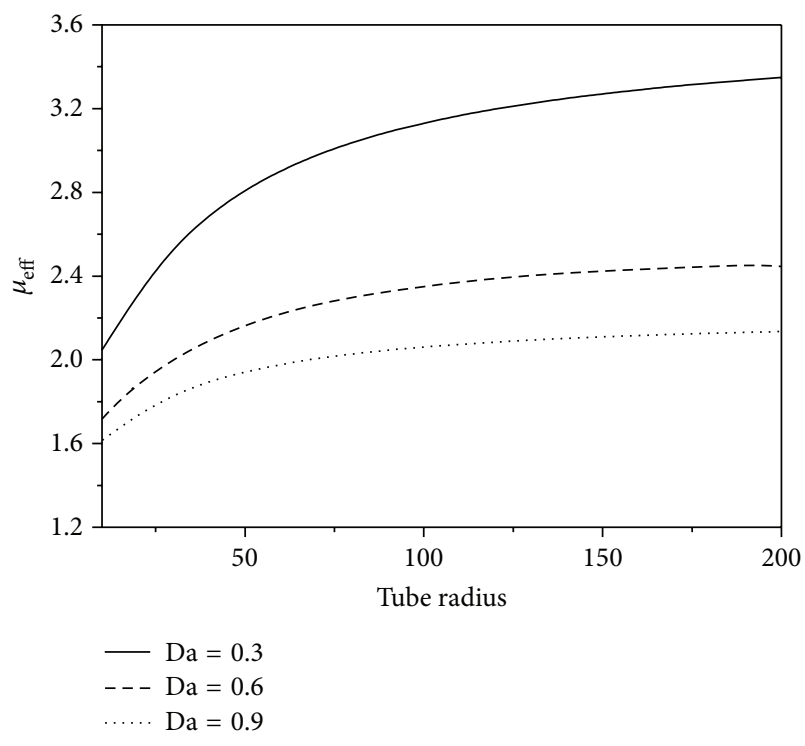

FIGURE 3: Effect of Da on $\mu_{\text {eff }}\left(H_{0}=40 \%, \lambda_{1}=2, M_{p}=1\right.$, and $M_{c}=$ $0.5)$.

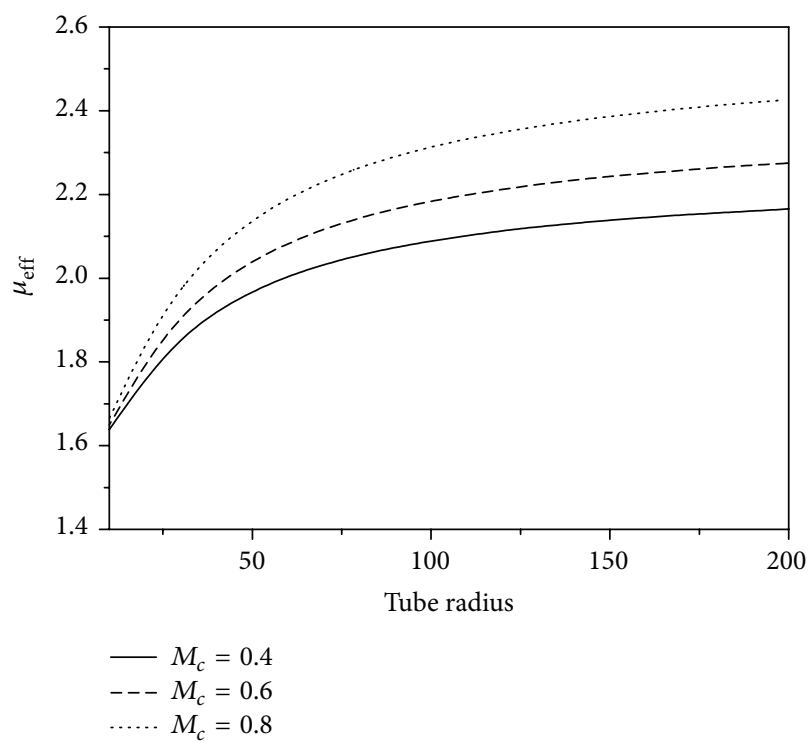

FIGURE 4: Effect of $M_{c}$ on $\mu_{\text {eff }}\left(H_{0}=40 \%, \lambda_{1}=2, M_{p}=1\right.$, and $\mathrm{Da}=$ 0.5 ).

[5]. Further, for given values of all the parameters, the effective viscosity $\left(\mu_{\text {eff }}\right)$ increases with tube radius $(a)$; that is, the flow exhibits the Fahraeus-Lindquist effect. For higher values of Jeffrey parameter $\left(\lambda_{1}\right)$ and Darcy number $(\mathrm{Da})$, the increase in effective viscosity with tube radius is not very significant for values of tube radius larger than $75 \mu \mathrm{m}$ (Figures 2 and 3 ).

The effects of Jeffrey parameter $\left(\lambda_{1}\right)$, core magnetic parameter $\left(M_{c}\right)$, Darcy number $(\mathrm{Da})$, tube hematocrit $\left(H_{0}\right)$, and tube radius $(a)$ on core hematocrit $\left(\bar{H}_{c}\right)$ and mean hematocrit $\left(\bar{H}_{m}\right)$ are depicted in Figures $6-13$. It can be seen that the core hematocrit $\left(\bar{H}_{c}\right)$ decreases with Jeffrey

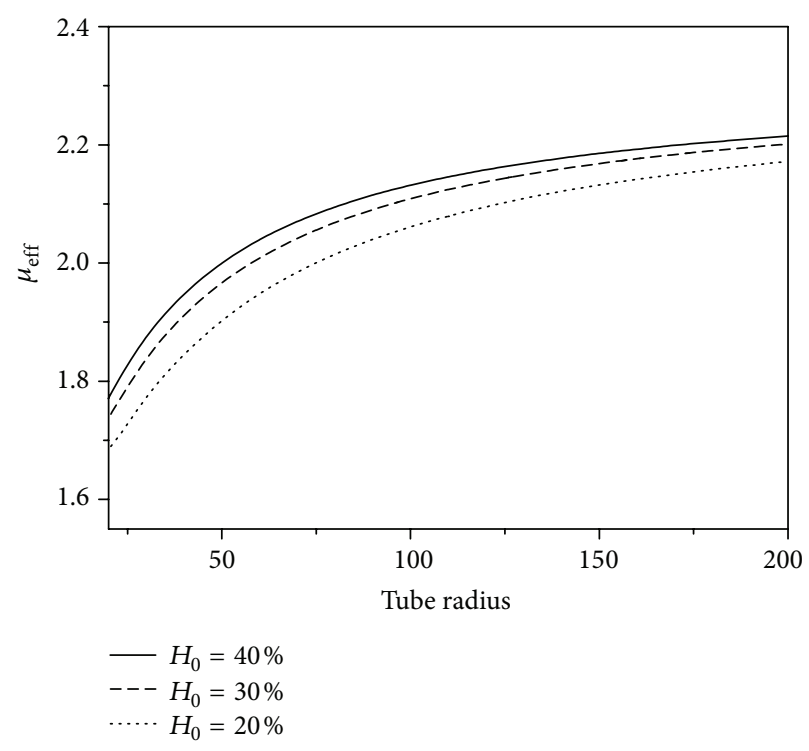

Figure 5: Effect of $H_{0}$ on $\mu_{\text {eff }}\left(\lambda_{1}=2, M_{p}=1, M_{c}=0.5\right.$, and $\mathrm{Da}=$ $0.5)$.

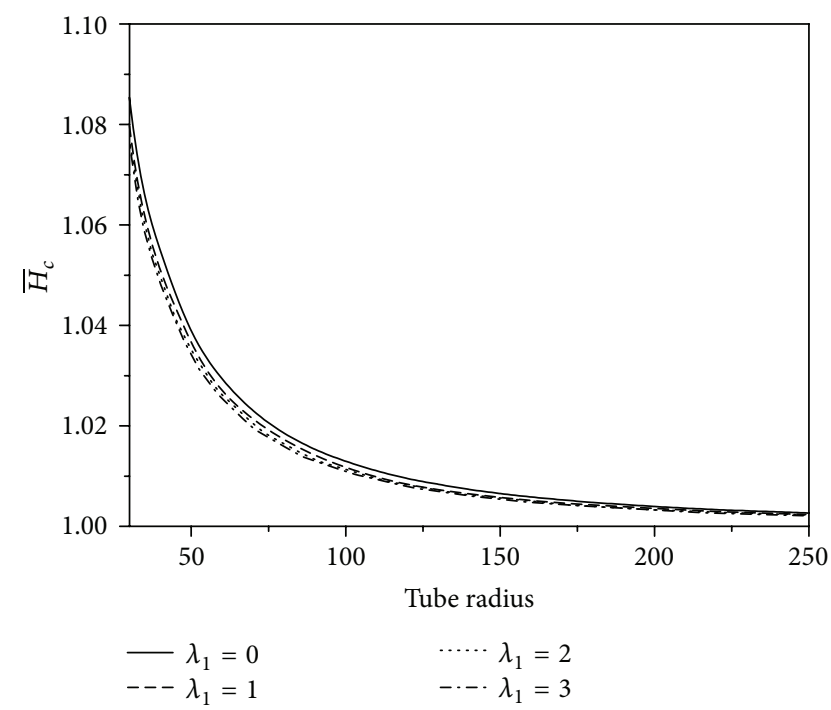

Figure 6: Effect of $\lambda_{1}$ on $\bar{H}_{c}\left(H_{0}=40 \%, M_{p}=1, M_{c}=0.5\right.$, and Da $=0.5)$.

parameter $\left(\lambda_{1}\right)$ (Figure 6), Darcy number (Da) (Figure 7), tube hematocrit $\left(H_{0}\right)$ (Figure 8 ), and tube radius " $a$." It can be observed that core hematocrit $\left(\bar{H}_{c}\right)$ decreases with tube radius (Figures 6-9). Figure 9 shows that the influence of magnetic parameter $\left(M_{c}\right)$ on core hematocrit is very insignificant. Also, the mean hematocrit $\left(\bar{H}_{m}\right)$ decreases with Jeffrey parameter $\left(\lambda_{1}\right)$ (Figure 10) and Darcy number (Da) (Figure 11) but increases with tube hematocrit $\left(H_{0}\right)$ (Figure 12) and tube radius " $a$." It can be observed that mean hematocrit $\left(\bar{H}_{m}\right)$ increases with tube radius (Figures 1013). The influence of magnetic parameter $\left(M_{c}\right)$ on mean hematocrit $\left(\bar{H}_{m}\right)$ is very insignificant (Figure 13 ). 


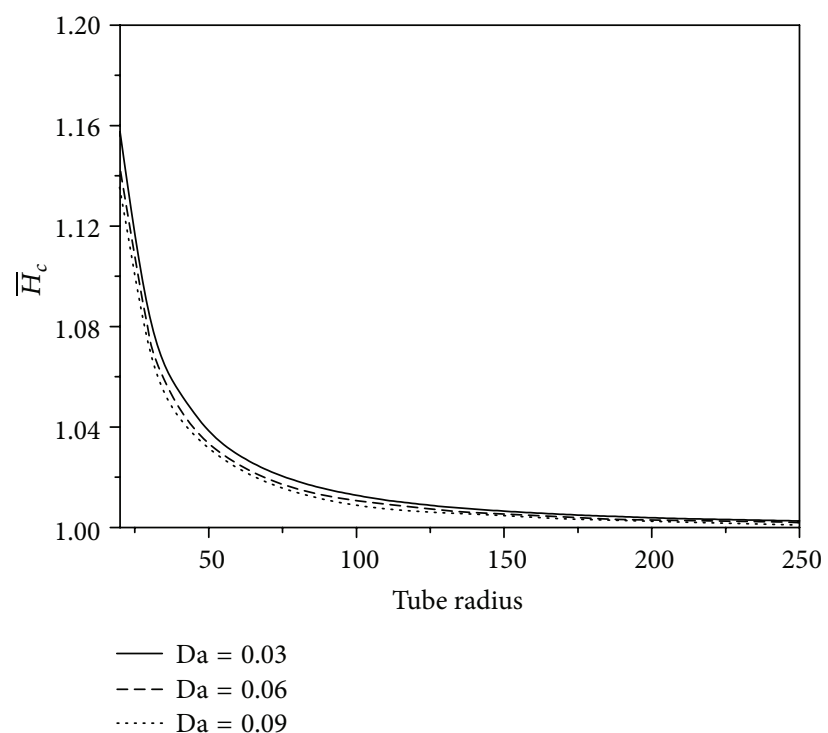

Figure 7: Effect of Da on $\bar{H}_{c}\left(H_{0}=40 \%, \lambda_{1}=2, M_{p}=1\right.$, and $M_{c}=$ $0.5)$.

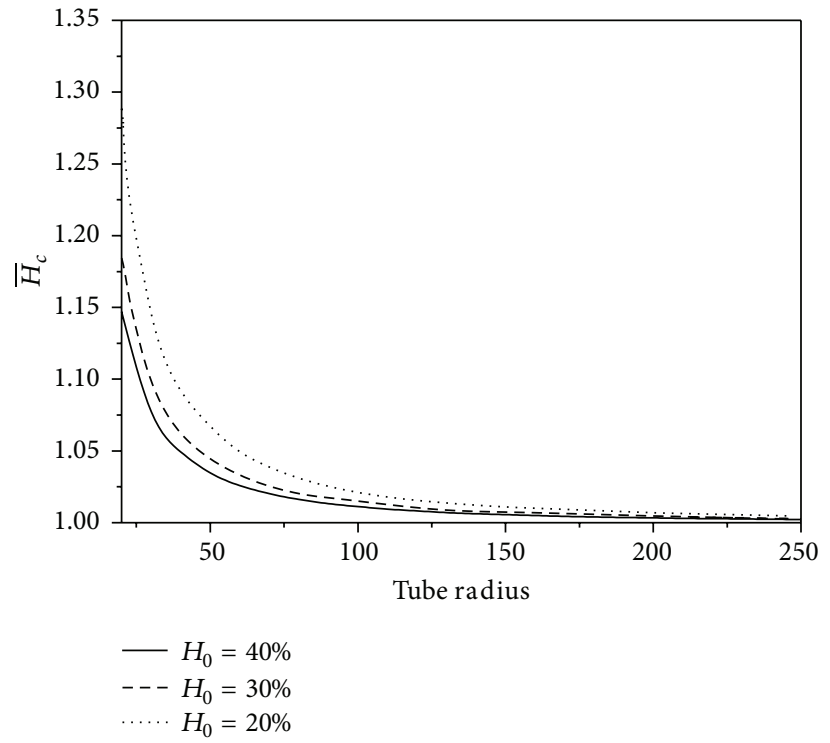

Figure 8: Effect of $H_{0}$ on $\bar{H}_{c}\left(\lambda_{1}=2, M_{p}=1, M_{c}=0.5\right.$, and $\mathrm{Da}=$ $0.5)$.

\section{Conclusions}

A two-fluid model has been proposed to describe flow through porous medium in small diameter tubes with Jeffrey fluid in the core region and Newtonian fluid in the peripheral region in the presence of a magnetic field. It is found that the effective viscosity increases with Jeffrey parameter, core magnetic parameter, Darcy number, tube hematocrit, and tube radius. Further, the core hematocrit decreases with Jeffrey parameter, Darcy number, tube hematocrit, and tube radius and the mean hematocrit increases with Jeffrey parameter, Darcy number, tube hematocrit, and tube radius.

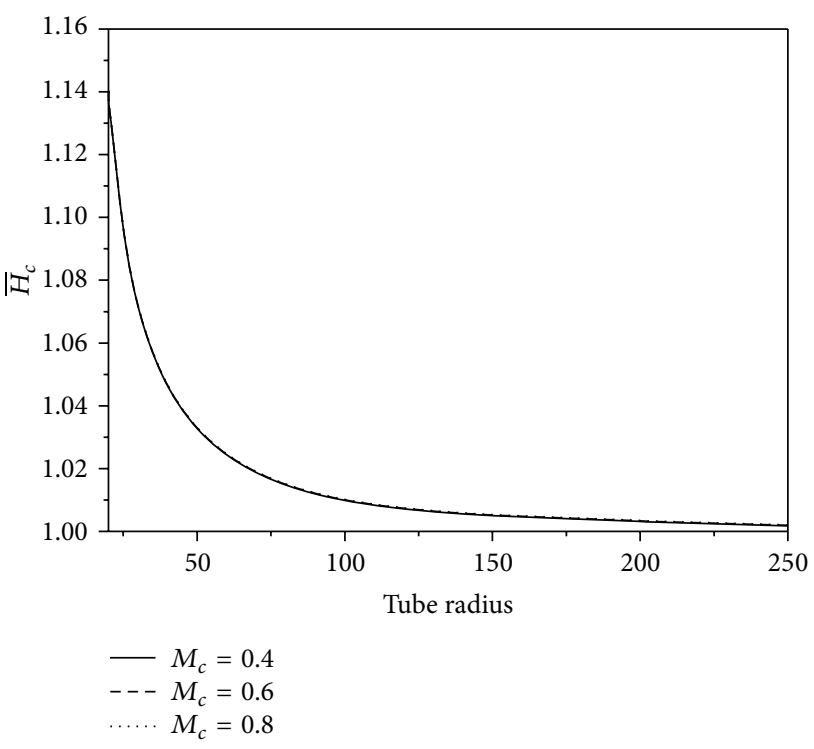

Figure 9: Effect of $M_{c}$ on $\bar{H}_{c}\left(H_{0}=40 \%, M_{p}=1, \lambda_{1}=2\right.$, and $\mathrm{Da}=$ $0.5)$.

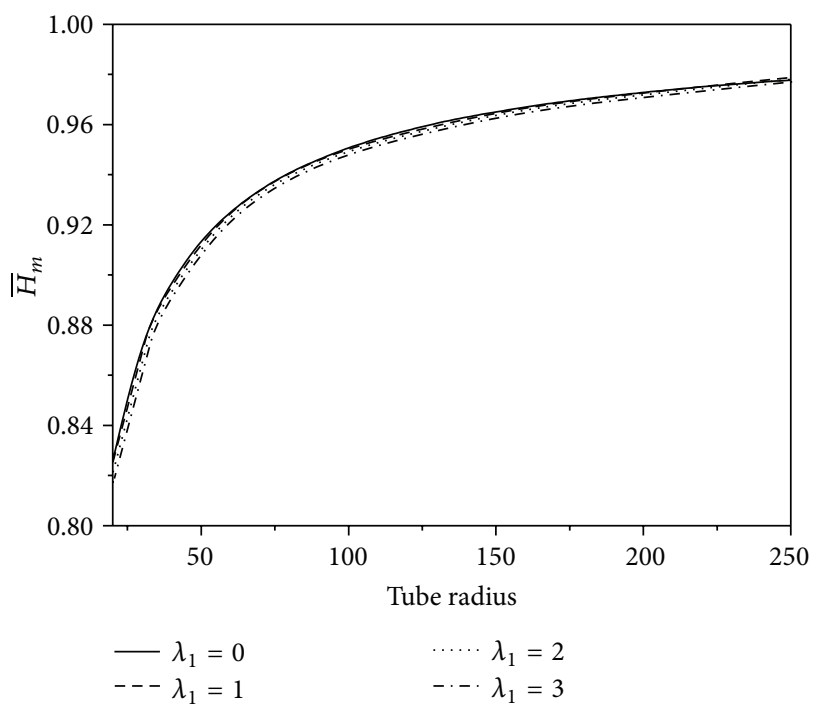

Figure 10: Effect of $\lambda_{1}$ on $\bar{H}_{m}\left(H_{0}=40 \%, M_{p}=1, M_{c}=0.5\right.$, and Da $=0.5$ ).

The influence of magnetic parameter on core hematocrit and mean hematocrit is very insignificant.

\section{Nomenclature}

a: Tube radius

$b$ : Radius of the core region

$d$ : Dimensionless core radius $=1-(\epsilon / a)$

$\epsilon:$ Peripheral region thickness $=a-b$

$\xi$ : Dimensionless radial direction $r / a$

$\mu^{\prime}$ : Ratio of viscosity $=\mu_{p} / \mu_{c}$

$\mu_{p}$ : Peripheral region viscosity 


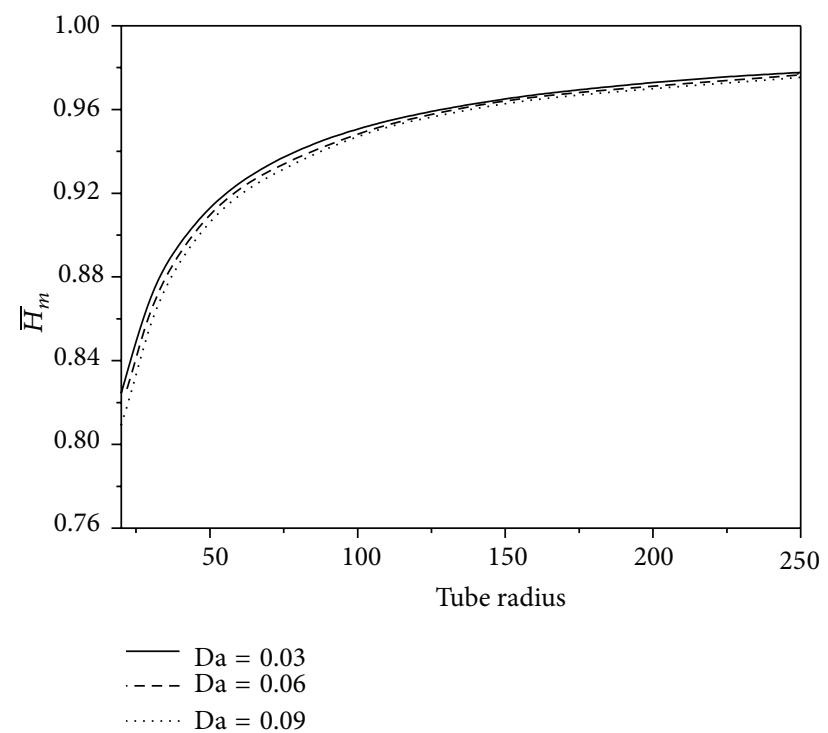

Figure 11: Effect of Da on $\bar{H}_{m}\left(H_{0}=40 \%, \lambda_{1}=2, M_{p}=1\right.$, and $M_{c}=$ $0.5)$.

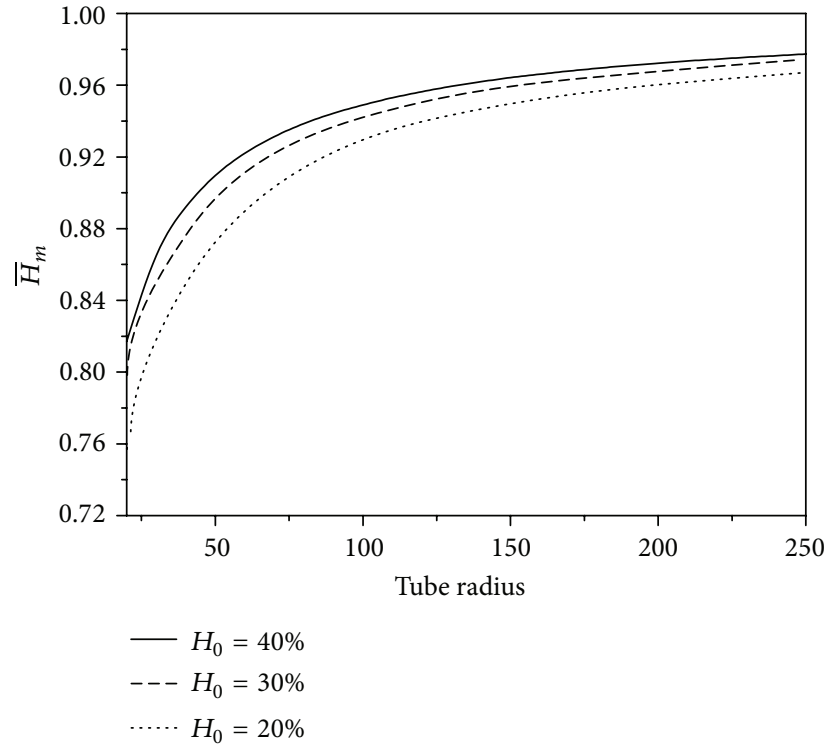

Figure 12: Effect of $H_{0}$ on $\bar{H}_{m}\left(\lambda_{1}=2, M_{p}=1, M_{c}=0.5\right.$, and $\mathrm{Da}=$ $0.5)$.

$\lambda_{1}:$ Jeffrey parameter

Da: Darcy number $=k_{0} / a^{2}$

$\sigma$ : $\quad$ Electrical conductivity

$M_{p}$ : Magnetic parameter in peripheral region $=\sqrt{\sigma / \mu_{p}} B_{0} a$

$M_{c}:$ Magnetic parameter in core region $=$ $\sqrt{\sigma / \mu_{c}} B_{0} a$

$r$ : Radial direction

$z$ : Axial direction

$v_{1}$ : Velocity in peripheral region

$v_{2}$ : Velocity in core region

$p$ : Pressure

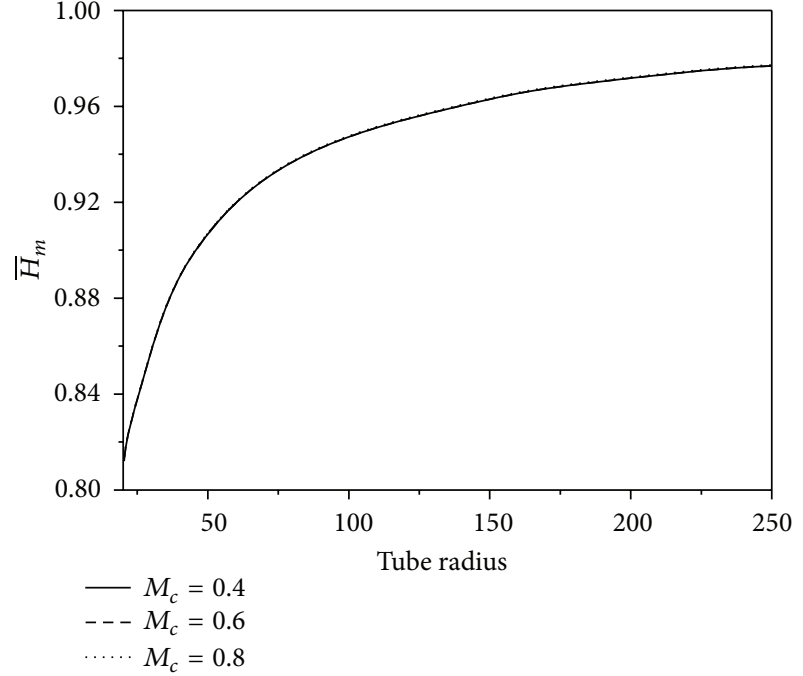

Figure 13: Effect of $M_{c}$ on $\bar{H}_{m}\left(H_{0}=40 \%, M_{p}=1, \lambda_{1}=2\right.$, and $\mathrm{Da}=$ $0.5)$.

$P: \quad$ Constant pressure gradient $=-(\partial p / \partial z)$

$\mu_{c}$ : Core region viscosity

$\rho$ : Density

$k_{0}$ : Permeability of porous medium

$B_{0}$ : Uniform magnetic field.

\section{Conflict of Interests}

The authors declare that there is no conflict of interests regarding the publication of this paper.

\section{Acknowledgment}

The authors thank the reviewers for their constructive suggestions which led to definite improvement in the paper.

\section{References}

[1] R. H. Haynes, "Physical basis of the dependence of blood viscosity on tube radius," The American Journal of Physiology, vol. 198, pp. 1193-1200, 1960.

[2] G. Bugliarello and J. Sevilla, "Velocity distribution and other characteristics of steady and pulsatile blood flow in fine glass tubes," Biorheology, vol. 7, no. 2, pp. 85-107, 1970.

[3] M. Sharan and A. S. Popel, "A two-phase model for flow of blood in narrow tubes with increased effective viscosity near the wall," Biorheology, vol. 38, no. 5-6, pp. 415-428, 2001.

[4] V. P. Srivastava, "A theoretical model for blood flow in small vessels," Applications and Applied Mathematics, vol. 2, no. 1, pp. 51-65, 2007.

[5] P. Chaturani and V. S. Upadhya, "On micropolar fluid model for blood flow through narrow tubes," Biorheology, vol. 16, no. 6, pp. 419-428, 1979.

[6] P. Chaturani, V. S. Upadhya, and S. P. Mahajan, "A two-fluid model for blood flow through small diameter tubes with nonzero couple stress boundary condition at interface," Biorheology, vol. 18, no. 2, pp. 245-253, 1981. 
[7] R. Bali and U. Awasthi, "Mathematical model of blood flow in small blood vessel in the presence of magnetic field," Applied Mathematics, vol. 2, no. 2, pp. 264-269, 2011.

[8] A. Kumar, C. L. Varshney, and G. C. Sharma, "Computational technique for flow in blood vessels with porous effects," Applied Mathematics and Mechanics, vol. 26, no. 1, pp. 63-72, 2005.

[9] A. K. Gupta, "Computational study of blood flow through stenosed artery with magnetic effects," International Journal of Information and Computation Technology, vol. 2, no. 1, pp. 8184, 2012.

[10] J. C. Misra, A. Sinha, and G. C. Shit, "Mathematical modeling of blood flow in a porous vessel having double stenoses in the presence of an external magnetic field," International Journal of Biomathematics, vol. 4, no. 2, pp. 207-225, 2011.

[11] N. T. M. Eldabe, B. M. Agoor, and H. Alame, "Peristaltic motion of non-newtonian fluid with heat and mass transfer through a porous medium in channel under uniform magnetic field," Journal of Fluids, vol. 2014, Article ID 525769, 12 pages, 2014.

[12] T. Hayat, N. Ali, S. Asghar, and A. M. Siddiqui, "Exact peristaltic flow in tubes with an endoscope," Applied Mathematics and Computation, vol. 182, no. 1, pp. 359-368, 2006.

[13] A. Ebaid, E. F. Elshehawey, N. T. Eldabe, and E. M. Elghazy, "Peristaltic transport in an asymmetric channel through a porous medium," Applied Mathematics and Computation, vol. 182, no. 1, pp. 140-150, 2006.

[14] K. Vajravelu, S. Sreenadh, and P. Lakshminarayana, "The influence of heat transfer on peristaltic transport of a Jeffrey fluid in a vertical porous stratum," Communications in Nonlinear Science and Numerical Simulation, vol. 16, no. 8, pp. 3107-3125, 2011.

[15] K. L. Jyothi, P. Devaki, and S. Sreenadh, "Pulsatile flow of a Jeffrey fluid in a circular tube having internal Porous lining," International Journal of Mathematical Archive, vol. 4, pp. 75-82, 2013.

[16] A. Ebaid, "Effects of magnetic field and wall slip conditions on the peristaltic transport of a Newtonian fluid in an asymmetric channel," Physics Letters A, vol. 372, no. 24, pp. 4493-4499, 2008.

[17] N. S. Akbar, S. Nadeem, and C. Lee, "Characteristics of Jeffrey fluid model for Peristaltic flow of Chyme," Results in Physics, vol. 3, pp. 152-160, 2013.

[18] A. M. Abd-Alla, S. M. Abo-Dahab, and M. M. Albalawi, "Magnetic field and gravity effects on peristaltic transport of a Jeffrey fluid in an asymmetric channel," Abstract and Applied Analysis, vol. 2014, Article ID 896121, 11 pages, 2014.

[19] S. Nallapu and G. Radhakrishnamacharya, "Flow of Jeffrey fluid through narrow tubes," International Journal of Scientific and Engineering Research, vol. 4, pp. 468-473, 2013. 


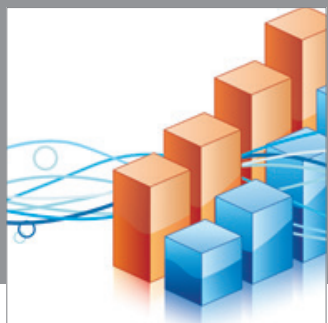

Advances in

Operations Research

mansans

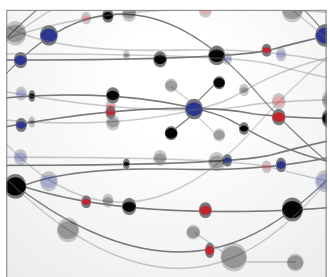

The Scientific World Journal
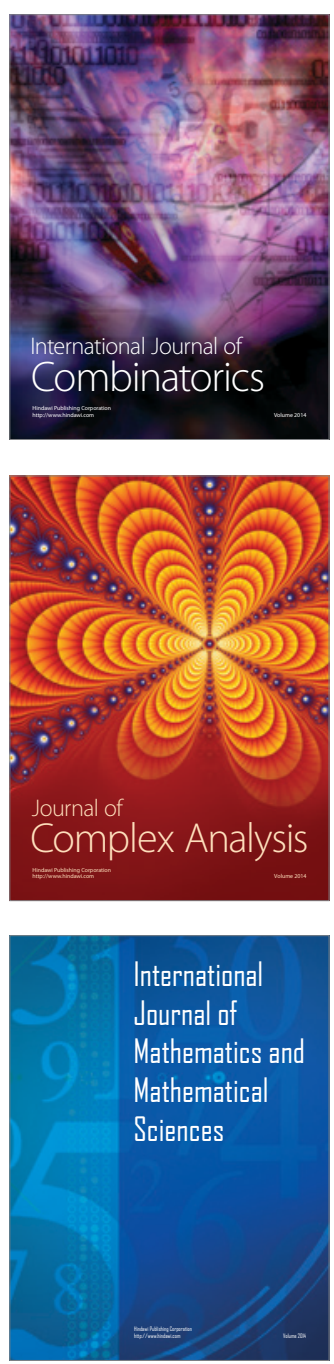
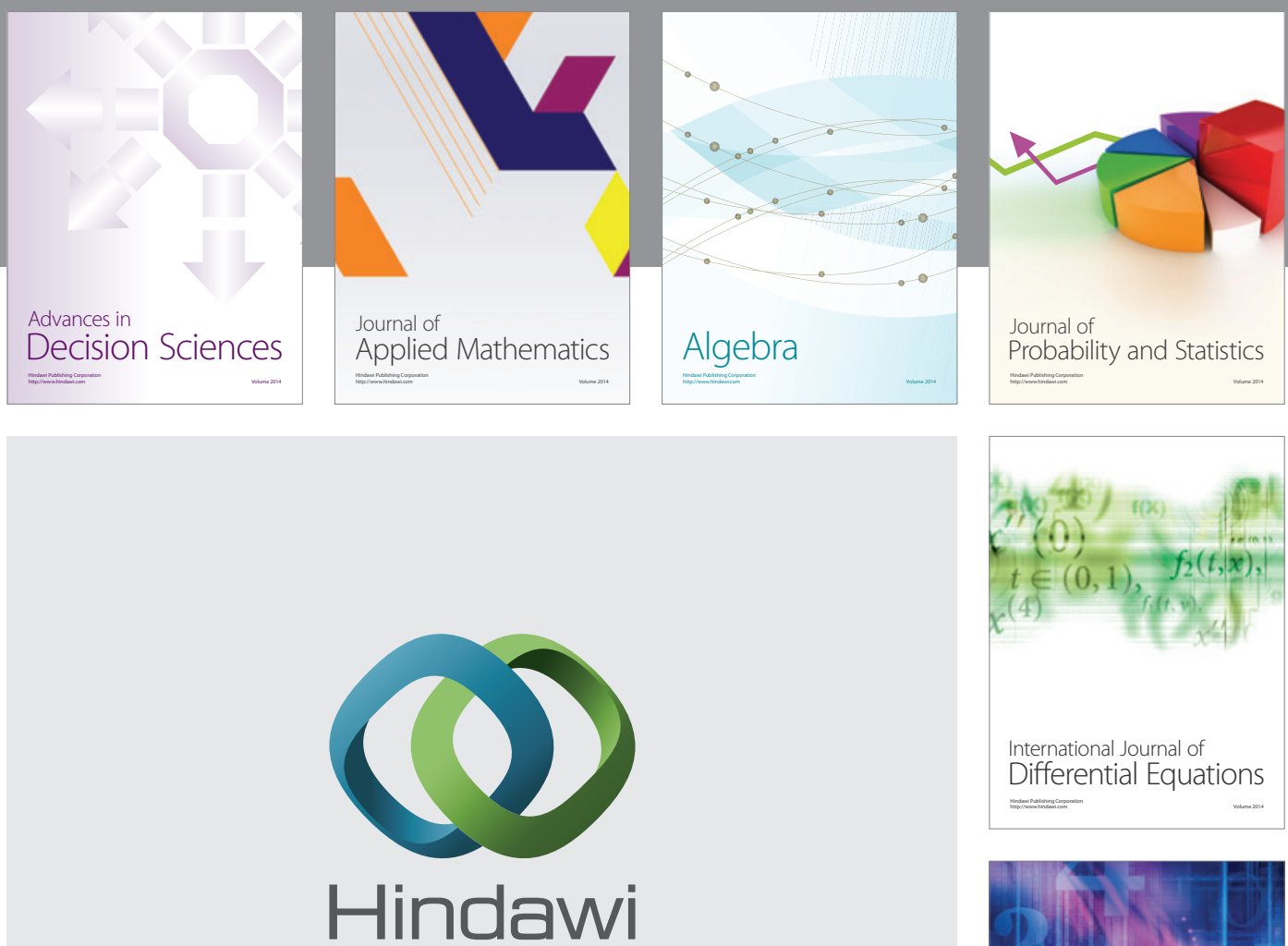

Submit your manuscripts at http://www.hindawi.com
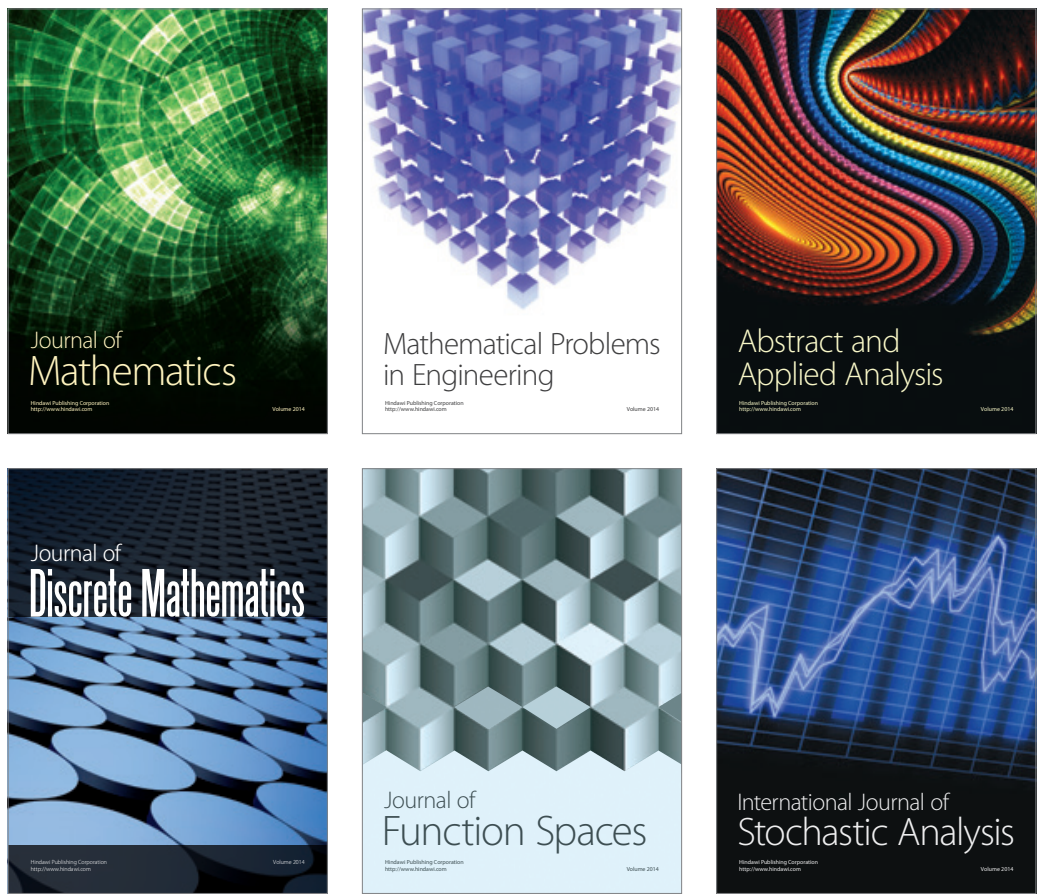

Journal of

Function Spaces

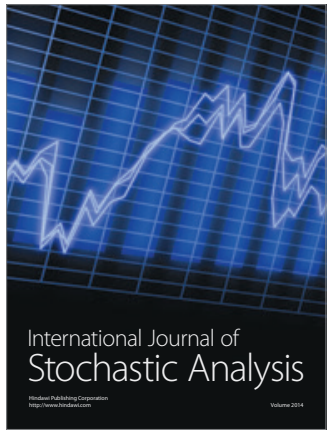

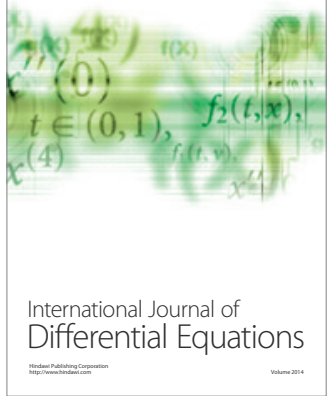
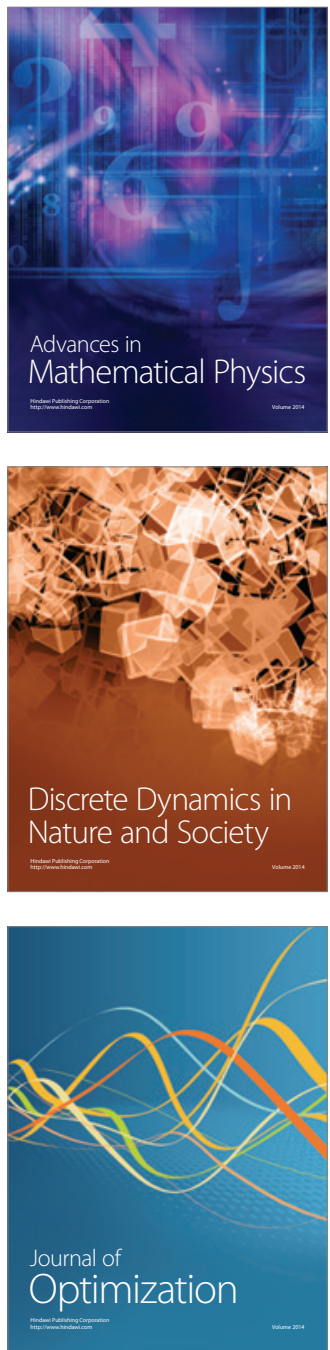